\title{
Diagnostic Significance of Cellular Neuroglial Tissue in Ovarian Immature Teratoma
}

Yun Chai · Chang Gok Woo Joo-Young Kim² . Chong Jai Kim Shin Kwang Khang · Jiyoon Kim In Ah Park · Eun Na Kim Kyu-Rae Kim

Department of Pathology, Asan Medical Center, University of Ulsan College of Medicine, Seoul; 'Department of Pathology, Soonchunhyang University Bucheon Hospital, Soonchunhyang University College of Medicine, Bucheon; 2Department of Pathology, Korea University Anam Hospital, Korea University College of Medicine, Seoul, Korea

Received: July 4, 2016

Revised: August 30, 2016

Accepted: September 19, 2016

\section{Corresponding Author}

Kyu-Rae Kim, MD, PhD

Department of Pathology, Asan Medical Center,

University of Ulsan College of Medicine, 88

Olympic-ro 43-gil, Songpa-gu, Seoul 05505, Korea

Tel: +82-2-3010-4514

Fax: $+82-2-472-7898$

E-mail:krkim@amc.seoul.kr
Background: Immature teratoma (IT) is a tumor containing immature neuroectodermal tissue, primarily in the form of neuroepithelial tubules. However, the diagnosis of tumors containing only cellular neuroglial tissue (CNT) without distinct neuroepithelial tubules is often difficult, since the histological characteristics of immature neuroectodermal tissues remain unclear. Here, we examined the significance of CNT and tried to define immature neuroectodermal tissues by comparing the histological features of neuroglial tissues between mature teratoma (MT) and IT. Methods: The histological features of neuroglial tissue, including the cellularity, border between the neuroglial and adjacent tissues, cellular composition, mitotic index, Ki-67 proliferation rate, presence or absence of tissue necrosis, vascularity, and endothelial hyperplasia, were compared between 91 MT and 35 IT cases. Results: CNTs with a cellularity grade of $\geq 2$ were observed in $96 \%$ of IT cases and $4 \%$ of MT cases $(p<.001)$; however, CNT with a cellularity grade of 3 in MT cases was confined to the histologically distinct granular layer of mature cerebellar tissue. Moreover, CNT in IT exhibited significantly higher rates of Ki-67 proliferation, mitoses, and necrosis than those in MT $(p<.001)$. Furthermore, an infiltrative border of neuroglial tissue and glomeruloid endothelial hyperplasia were significantly more frequent in IT cases than in MT cases $(p<.001)$. Conclusions: Our results suggest that if CNT with a cellularity grade of $\geq 2$ is not a component of cerebellar tissue, such cases should be diagnosed as IT containing immature neuroectodermal tissue, particularly if they exhibit an infiltrative border, mitoses, necrosis, and increased Ki-67 proliferation.

Key Words: Immature teratoma; Neuroectodermal; Neuroglia; Neuroepithelium; Ki-67; Ovary
Immature teratoma (IT) is the second most common malignant germ-cell tumor of the ovary, and accounts for $<1 \%$ of all ovarian teratomas. Although the identification of the immature neuroectodermal component is important in the diagnosis and grading of IT, the histological definition of immature neuroectodermal tissue is ambiguous, regardless of whether it applies only to immature neuroepithelial tubules/rosettes or includes other types of immature neuroectodermal tissue, such as cellular neuroglial tissue (CNT). In some cases of CNT only, without any distinct immature neuroepithelial tubules/rosettes in the background of the mature teratomatous elements, the diagnosis is very difficult, particularly in cases where frozen sections are used (Fig. 1).

In the present study, we aimed to define the histological criteria of immature neuroectodermal tissue and examine the significance of CNT by comparing the histological features of neuroglial tissues between IT and mature teratoma (MT).

\section{MATERIALS AND METHODS}

\section{Patient selection}

Cases of IT and MT treated between January 1989 and December 2012, including neuroglial tissue of the ovary, were assessed from the database of the Department of Pathology, Asan Medical Center, Seoul, Korea. Of 70 cases of ovarian IT diagnosed during this period, 13 were excluded due to the presence of a mixed germ-cell tumor component and 22 were excluded due to unavailability of a specimen for slide review or because of the lack of distinct immature neuroepithelial tubules or rosettes. However, the histopathological review in the present study focused only on the areas of CNT. The diagnosis of IT in this study was 
based on the presence of immature neuroepithelial tubules or rosettes within the tumor, regardless of the presence or absence of CNT; 35 IT cases and 91 mature cystic teratoma (MT) cases

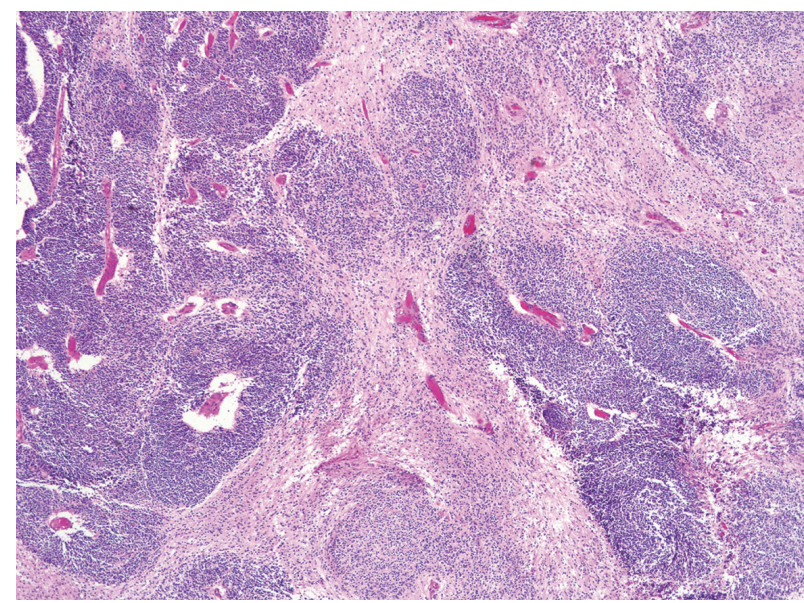

Fig. 1. Cellular neuroglial tissue. The diagnosis of immature teratoma containing only cellular neuroglial tissue without distinct neuroepithelial tubules is often difficult since the histological characteristics of immature neuroectodermal tissues have not been clearly defined.
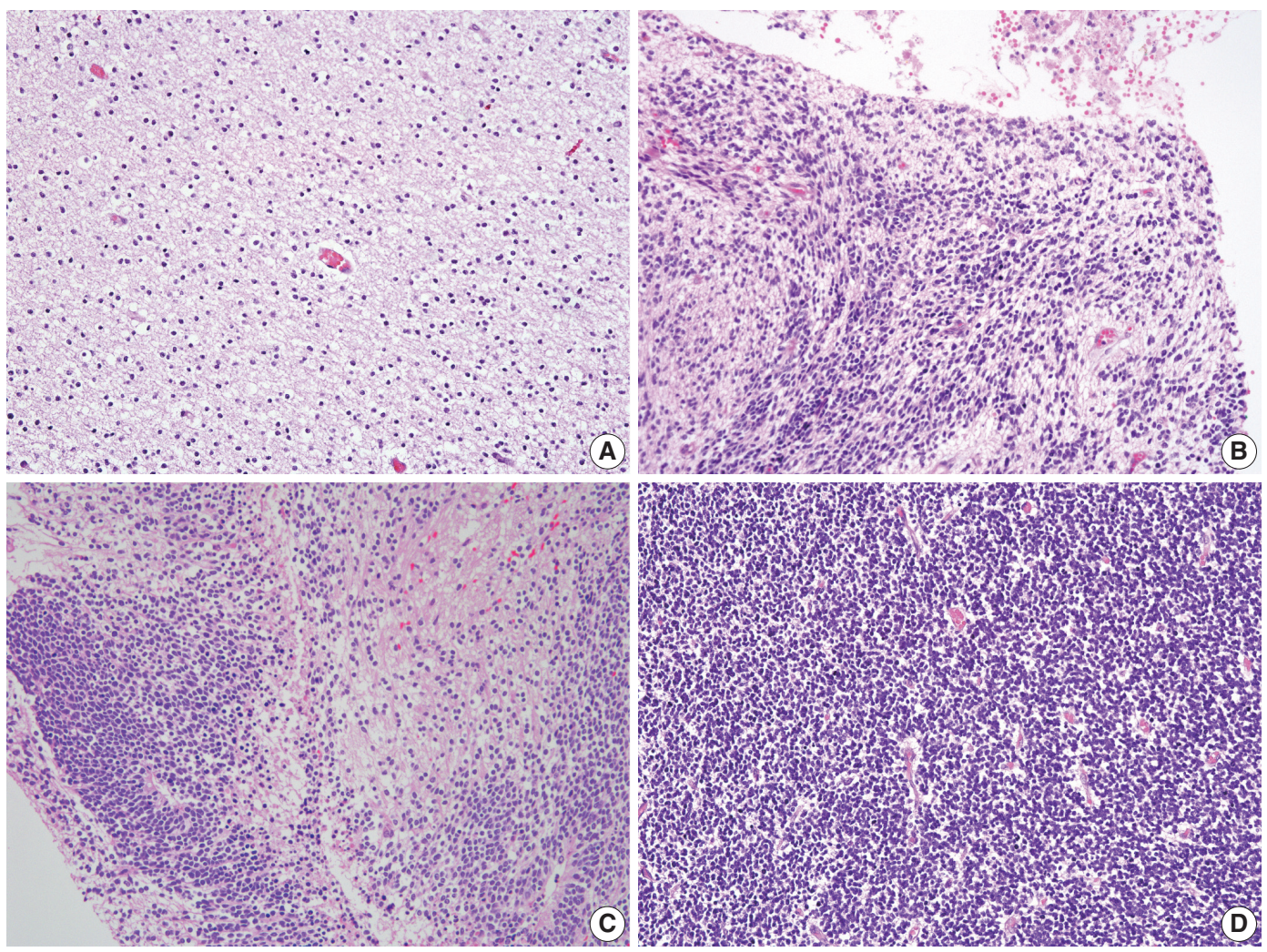

Fig. 2. Cellularity of neuroglial tissue in mature and immature teratomas. (A) Grade 1 referred to cellularity comparable to that of normal white matter or an inter-nuclear distance longer than 5 nuclear diameters. (B, C) Grade 2 applied to cases with either heterogeneous cellularity or with inter-nuclear distances similar to 2-4 nuclear diameters. (D) Grade 3 cellularity referred to cellularity comparable to that of the germinal matrix of the fetal brain or the granular layer of the normal adult cerebellum wherein nuclei touch each other, or an inter-nuclear distance shorter than 1 nuclear diameter. with a neuroglial component were finally included in this study. All of the tissue sections were formalin-fixed and paraffin-embedded. Hematoxylin and eosin-stained slides were available for review in all cases. The Institutional Review Board of the University of Ulsan College of Medicine, Asan Medical Center, Seoul, Korea, approved the collection and use of the samples in this study for research purposes (protocol number: S2016-10600001).

\section{Histological evaluation}

Histopathological findings were reviewed by two pathologists blinded to the diagnosis (Y.C. and K-R.K.). We compared the histological features of the neuroglial components between the two groups (IT and MT). The histologic features examined included cellularity of the neuroglial tissue, border between the neuroglial and surrounding non-neuroglial tissues, cellular component within the CNT (either polymorphous or monomorphous), average number of mitoses per high-power $(\times 400)$ field, presence or absence of necrosis, vascular proliferation, and glomeruloid endothelial hyperplasia. 

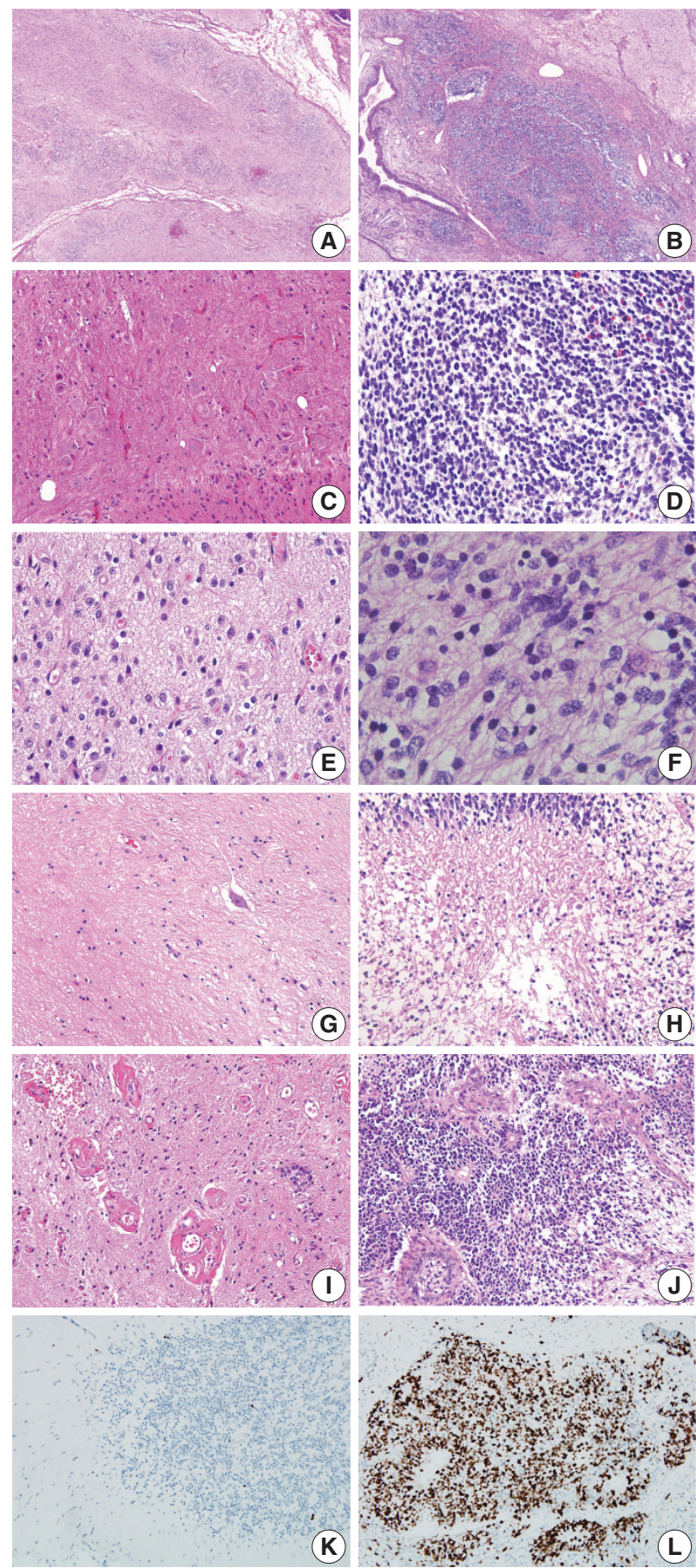

Fig. 3. Histopathologic features of mature teratoma and immature teratoma. Neuroglial cells in a mature teratoma show a smooth bor$\operatorname{der}(\mathrm{A})$, and comprise polymorphous cellular components (C). They show no mitotic activity (E) and no coagulative necrosis (G), but do exhibit focally glomeruloid endothelial proliferation (I) and rare Ki-67positive proliferating cells (K). In contrast, cellular neuroglial cells in immature teratoma show an infiltrative border (B) more frequently, and comprise monomorphic germinal matrix-like cells (D). They also show frequent mitoses $(F)$, coagulative necrosis $(H)$, glomeruloid endothelial proliferation (J), and increased Ki-67-positive proliferating cells (L).
We selected the areas of highest cellularity within the tumor; cellularity was classified into three grades according to the density of the cellular component (Fig. 2). Grade 1 referred to cellularity comparable to that of normal white matter, or with an inter-nuclear distance longer than five nuclear diameters (Fig. 2A). Grade 2 applied to cases with either heterogeneous cellularity or with inter-nuclear distances corresponding to $2-4$ nuclear diameters in the area with the highest cellularity (Fig. 2B, C). Grade 3 was assigned to cellularity similar to that of the germinal matrix of the fetal brain or granular layer of the normal adult cerebellum wherein the nuclei touch each other, or with an inter-nuclear distance shorter than 1 nuclear diameter (Fig. 2D).

The border status was divided into an infiltrative or pushing border (Fig. 3A, B). The infiltrative border (Fig. 3B) was assigned to cases with an irregular border, in contrast to the pushing border (Fig. 3A), which exhibited a smooth and well-circumscribed edge. The cellular component within the neuroglial tissue was categorized as polymorphous or monomorphous (Fig. 3C, D). When neuroglial tissue was composed of round, neuroblast-like cells and spindle or stellate-shaped glial cells, it was classified as polymorphous (Fig. 3C); when the tissue was uniformly composed of monotonous small and round to ovoid cells, it was categorized as monomorphous (Fig. 3D).

The mitotic score groups were established according to the average number of mitotic figures per high-power field, as score 1 (no mitotic activity), score 2 ( $1-4$ mitoses per high-power field), score 3 (5-9 mitoses per high-power field), or score 4 ( $\geq 10 \mathrm{mi}$ toses per high-power field) (Fig. 3E, F). The presence or absence of coagulative necrosis (Fig. 3G, H), vascular proliferation, and glomeruloid endothelial hyperplasia (Fig. 3I, J) was also recorded.

The presence of vascular proliferation in CNT was determined based on the number of small blood vessels in a high-power field, compared to that in the normal cerebral cortex. Glomeruloid endothelial hyperplasia was defined as compact stratification of proliferating endothelial and perithelial (smooth muscle) cells that resembles a renal glomerulus, similar to that observed in glioblastoma multiforme.

\section{Statistical analysis}

The differences between the two groups were analyzed using the chi-square test, Fisher exact test, and Mann-Whitney U test. A two-sided p-value $<0.05$ was considered statistically significant. All statistical calculations were performed using the SPSS ver. 18.0 (SPSS Inc., Chicago, IL, USA). 
Table 1. Demographic data for the study patients with mature and immature teratomas of the ovary

\begin{tabular}{lccc}
\hline Variable & MT & IT & p-value \\
\hline Age (yr) & $28.9(3-67)$ & $20.6(5-36)$ & $<.001$ \\
Tumor size (cm) & $7.2(1.2-16.5)$ & $13.6(2-26)^{\mathrm{a}}$ & $<.001$ \\
Surgical procedure & & & $<.001$ \\
$\quad$ Unilateral oophorectomy & $9(9.9)$ & $21(61.8)$ \\
$\quad$ Bilateral oophorectomy & $2(2.2)$ & $5(14.7)$ \\
$\quad$ Unilateral or bilateral cystectomy & $76(83.5)$ & $4(11.8)$ \\
$\quad$ Unilateral oophorectomy+contralateral cystectomy & $4(4.4)$ & $4(11.8)$ \\
Laterality & $74(81.3)$ & $33(97.1)$ \\
$\quad$ Unilateral & & $3(8.8)$ & .026 \\
$\quad+$ Contralateral mature cystic teratoma & & $1(2.9)$ & $1(2.9)^{b}$ \\
$\quad$ Contralateral mixed germ-cell tumor & $17(18.7)$ &
\end{tabular}

Values are presented as mean (range) or number (\%).

MT, mature teratoma; IT, immature teratoma.

${ }^{a}$ Tumor size was evaluated only in 26 cases of IT; ${ }^{b}$ This patient experienced recurrence in the opposite ovary 21 years after surgery.

Table 2. Demographic data for the study patients with immature teratoma of the ovary

\begin{tabular}{lc}
\hline Variable & Immature teratoma (\%) \\
\hline Tumor grade & $9(25.7)$ \\
Grade 1 & $16(45.7)$ \\
Grade 2 & $10(28.6)$ \\
Grade 3 & \\
Increased serum levels of tumor markers & $14(40.0)$ \\
AFP & $1(2.8)$ \\
$\beta$-hCG & $1(2.8)$ \\
CEA & $17(48.6)$ \\
CA125 & $6(17.1)$ \\
CA19-9 & \\
FIGO stage & $21(60.0)$ \\
IA & $5(14.2)$ \\
IC & $1(2.9)$ \\
IIB & $7(20.0)$ \\
IIIA & $1(2.9)$ \\
IIIC & \\
Adjuvant chemotherapy & $30(85.7)$ \\
Present & $5(15.3)$ \\
Absent & \\
Overall survival & $34(97.2)$ \\
Alive & $1(2.8)$ \\
dead & \\
\hline
\end{tabular}

AFP, alpha-fetoprotein; $\beta$-hCG, beta-human chorionic gonadotropin; CEA, carcinoembryonic antigen; CA125, cancer antigen 125; CA19-9, carbohydrate antigen 19-9; FIGO, International Federation of Gynecology and Obstetrics.

\section{RESULTS}

\section{Histopathological characteristics of MT and IT}

The demographic data for 35 patients with ovarian IT (mean age, 20.6 years; range, 5 to 36 years) and MT (mean age, 28.9 years; range, 3 to 67 years) are summarized in Tables 1 and 2 . Nine cases of IT were excluded in the analysis of demographic data due to insufficient information. Among the remaining 26 cases, those with IT showed a significantly larger tumor size (range, 2 to $26 \mathrm{~cm}$; mean, $13.6 \mathrm{~cm}$ ) as compared to those with MT (range, 1.2 to $16.5 \mathrm{~cm}$; mean, $7.2 \mathrm{~cm}$ ). The modalities of surgical treatment differed significantly between the two groups. In the IT group, unilateral oophorectomy was performed in 21 cases $(61.8 \%)$, unilateral oophorectomy with contralateral cystectomy in four cases $(11.8 \%)$, and bilateral oophorectomy in five cases $(14.7 \%)$. Unilateral or bilateral cystectomy was used in only four cases $(11.8 \%)$ in the IT group but was employed in a large proportion of MT cases ( 76 cases, $83.5 \%$ ).

Bilaterality was observed in only one case of IT $(2.9 \%)$ in contrast to $18.7 \%$ in mature cystic teratoma cases. In the case with bilateral IT, the patient had a recurrent tumor in the contralateral ovary 21 years after the first diagnosis. In three cases, IT was accompanied by mature cystic teratomas in the contralateral ovary. The serum tumor marker levels were within the normal range in almost half of the cases (15 cases, $42.9 \%$ ).

Higher serum levels of cancer antigen 125 in 17 patients (48.6\%), carbohydrate antigen $19-9$ in six patients (17.1\%), $\alpha$-fetoprotein in 14 patients $(40.0 \%), \beta$-human chorionic gonadotropin in one patient $(2.8 \%)$, and carcinoembryonic antigen in one patient $(2.8 \%)$ with IT were observed. Moreover, 21 patients had International Federation of Gynecology and Obstetrics (FIGO) stage IA, five had FIGO stage IC, one had FIGO stage IIB, seven had FIGO stage IIIA, and one had FIGO stage IIIC. Thirty patients $(85.7 \%)$ underwent postoperative adjuvant chemotherapy. All the patients with IT, except for one patient who died of sepsis during the chemotherapy, were alive without any tumor at the last follow-up. The patient with bilateral IT, who developed an IT in the opposite ovary, was also well without 
any tumor at the last follow-up.

\section{Histopathology of CNT}

All patients with IT showed CNT with heterogeneous cellularity throughout the tumor; grade 2 cellularity was noted in 18 patients $(51.4 \%)$ and grade 3 was noted in 17 patients $(48.6 \%)$ in addition to the glial tissue with grade 1 cellularity. However, all of the patients with MT, except for four cases, demonstrated uniform grade 1 cellularity, which resembled normal white matter (95.6\%). In the MT cases with CNT, the CNT was exclusively confined to the histologically distinct granular layer of mature cerebellar tissue, which could be recognized by the Purkinje cells. CNT, defined as neuroglial tissue with $\geq$ grade 2 cellularity, was significantly more frequent in IT than in MT cases $(\mathrm{p}<.001)$.

The infiltrative border was more frequent in IT than in MT cases, and the difference was statistically significant (74.3\% and $36.3 \%$, respectively; $\mathrm{p}<.001)$. The CNT in MT comprised a monomorphic cellular component, whereas the CNT in IT comprised polymorphous cells with varying morphology. Furthermore, vascular proliferation was more frequent in IT than in MT cases, although the difference was not statistically significant $(\mathrm{p}=.512)$. However, glomeruloid endothelial hyperplasia was significantly more frequent in IT than in MT cases $(28.6 \%$ and $2.2 \%$, respectively; $\mathrm{p}<.001$ ).

The difference between the numbers of mitotic figures in the two groups was significant (Fig. 3E, F). High mitotic activity ( $\geq$ 5 mitotic figures per high-power field) was found only in the IT group. Coagulation necrosis was observed only in IT cases, and all these cases had mitotic score groups of 3 or 4 with $>5$ mitoses per high-power field. The histopathological differences in the neuroglial tissue between the two groups are summarized in Table 3.

\section{DISCUSSION}

The diagnostic criteria of IT is somewhat ambiguous. At present, IT is defined either as a tumor containing immature or primitive immature neuroepithelial tubules or rosettes, ${ }^{1-4}$ or a tumor containing immature embryonal or fetal-type tissue. However, a more precise definition of IT is required for the differential diagnosis of pure IT from a pure yolk sac tumor or a mixed germ-cell tumor containing yolk sac tumor and IT components, as immature embryonal/fetal-type tissue other than the immature neuroectodermal tissue including immature enteric, hepatic, or endometrioid-like tissues is occasionally observed in yolk sac tumors.

The histopathological features of germ-cell tumors frequently

Table 3. Comparison of the histopathological features of the CNT between mature and immature teratoma

\begin{tabular}{|c|c|c|c|}
\hline Variable & Mature teratoma (\%) & Immature teratoma (\%) & $p$-value \\
\hline No. of cases & 91 & 35 & \\
\hline Cellularity of CNT & & & $<.001$ \\
\hline Grade 1 & $87(95.6)$ & 0 & \\
\hline Grade 2 & 0 & $18(51.4)$ & \\
\hline Grade 3 & $4(4.4)$ & 17 (48.6) & \\
\hline Border status & & & $<.001$ \\
\hline Pushing & $58(63.7)$ & $9(25.7)$ & \\
\hline Infiltrative & $33(36.3)$ & $26(74.3)$ & \\
\hline Cellular composition & & & $<.001$ \\
\hline Polymorphous & $91(100)$ & $26(74.3)$ & \\
\hline Monomorphic & 0 & $9(25.7)$ & \\
\hline Increased vascularity & $2(2.2)$ & 0 & .512 \\
\hline Glomeruloid endothelial hyperplasia & $2(2.2)$ & $10(28.6)$ & $<.001$ \\
\hline Score 1 (no mitosis) & $90(98.9)$ & $13(37.1)$ & \\
\hline Score 2 (1-4/HPF) & $1(1.1)$ & $14(40.0)$ & \\
\hline Score 3 (5-9/HPF) & 0 & $2(5.7)$ & \\
\hline Score 4 (> 10/HPF) & 0 & $6(17.1)$ & \\
\hline Necrosis & & & $<.001$ \\
\hline Present & 0 & $29(82.9)$ & \\
\hline Absent & $91(100)$ & $6(17.1)$ & \\
\hline
\end{tabular}

Mitoses were expressed as a mean number of mitoses per one high power field.

CNT, cellular neuroglial tissue; HPF, high-power field. 
recapitulate the normal developmental stages of the embryo/fetus. Dysgerminoma resembles undifferentiated germ cells, choriocarcinoma resembles the cells of a developing placenta, and IT resembles immature or developing brain tissue. Immature neuroepithelium is one of the few structures in the human body that disappears after the completion of fetal development, and only exists during the fetal period. Hence, it is reasonable to state that the diagnosis of IT should be limited to tumors containing immature neuroectodermal tissue rather than immature embryonal or fetal-type tissue.

It is important to accurately identify immature neuroectodermal tissue because the treatment and prognosis of patients with IT depend on the amount of immature neuroectodermal tissue, which also forms the basis of the histological grading systems of IT. ${ }^{6,7}$ The immature neuroepithelial tubules of IT mimic the early neural tube during the normal developmental stage of the central nervous system. As the neural tube closes, the pseudostratified columnar epithelium in the neural tube develops into the ependymal zone in the innermost zone around the ventricular lumen, containing dividing neuroepithelial cells; the mantle zone around the neuroepithelial layer, containing neuroblasts derived from neuroepithelial cells; and the outermost marginal zone, containing nerve fibers emerging from neuroblasts. The neuroepithelial cells in the ependymal zone rapidly proliferate via symmetric and asymmetric cell divisions, and give rise to ependymoblasts, which remain in the ventricular zone, as well as glioblasts and post-mitotic neurons, which migrate to the marginal zone where they continue to differentiate. ${ }^{8,9}$ The germinal matrix of the fetal brain is a highly cellular and vascularized area containing glioblasts and post-mitotic neurons, ${ }^{10-12}$ from which all the neurons and supporting cells (glial cells) actively migrate during brain development. Our preliminary study has shown that small and round cells included in the CNT of IT showed immunoreactivity for nestin - a useful marker for precursor cells of neuroectodermal and mesenchymal lineages (data not shown). Moreover, the CNT is composed of polymorphous cellular components resembling glioblasts and neuroblasts, which suggests that the CNT may represent immature neuroectodermal tissue resembling the germinal matrix of the fetal brain.

In our present study, the neuroglial tissue in mature cystic teratoma cases exhibited grade 1 cellularity in all but four cases (96\%). A review of the histopathological findings of the four cases indicated that the CNT in MT cases was exclusively confined to the well-developed and histologically distinct cerebellar tissue. The cellularity in the granular layer of the cerebellum in MT cases was almost indistinguishable from that of the CNT in IT cases; however, the number of mitotic figures differed significantly. Increased mitotic activity ( $\geq 5$ mitotic figures per high-power field) was only observed in IT cases, whereas the granular layer of the cerebellum did not show any mitotic figures. Moreover, necrosis was observed only in the CNT of IT cases. The Ki-67 proliferation rate was also very low in the cerebellar granular layer, indicating that these are terminally differentiated cells. The gray matter of the cerebellar cortex is composed of three layers: the molecular layer, Purkinje cell layer, and granular layer. The cells in the granular layer are smallest cells in the body $(4-5 \mu \mathrm{m}$ in diameter), and have monomorphous, small and round nuclei with a coarse chromatin pattern. In contrast, the CNT of IT cases comprises polymorphous cells, which are similar to the glioblasts and neuroblasts in the germinal matrix of the fetal brain. Thus, the CNT in MT cases can be distinguished from the CNT in IT cases based on the monomorphic cellular component; presence of Purkinje cells; and absence of mitoses, necrosis, or proliferating activity. Moreover, the neuroglial tissue in IT cases showed heterogeneous cellularity throughout the tumor, whereas the neuroglial tissue in MT cases had a relatively uniform cellularity.

The diagnosis of IT in our current study was solely based on the presence of neuroepithelial tubules/rosettes in the tumor; however, all IT cases contained CNT with a cellularity grade of $\geq 2$ in at least some areas within the tumors. Thus, CNT with a cellularity grade of $\geq 2$ appears to be a characteristic feature of IT. However, additional tissue sampling would increase the likelihood of detecting immature neuroepithelial tubules in other areas, which would aid in the diagnosis.

As blood supply plays an important role in tumor growth, ${ }^{13}$ we compared the presence or absence of coagulation necrosis, vascular proliferation, and glomeruloid endothelial hyperplasia between the two groups. Vascular proliferation and glomeruloid endothelial hyperplasia within neuroglial tissue are common features of malignant neuroglial tumors such as glioblastoma. ${ }^{13,14}$ The number of blood vessels within the neuroglial tissue was not significantly different between the MT and IT cases in the present study; however, glomeruloid endothelial hyperplasia, reminiscent of glioblastoma, was significantly more frequent in IT cases than in MT cases.

Based on these results, we conclude that if CNT with a cellularity grade of $\geq 2$ is not a component of mature cerebellar tissue, it may then represent an additional feature of ovarian IT. Higher rates of Ki-67 proliferation (Fig. 3K, L), mitoses, necrosis, infiltrative border of neuroglial tissue, polymorphous cellular compo- 
nent, and the presence of glomeruloid endothelial hyperplasia within the CNT are the supporting features indicating IT.

\section{Conflicts of Interest}

No potential conflict of interest relevant to this article was reported.

\section{REFERENCES}

1. Koulos JP, Hoffman JS, Steinhoff MM. Immature teratoma of the ovary. Gynecol Oncol 1989; 34: 46-9.

2. Gershenson DM, del Junco G, Silva EG, Copeland LJ, Wharton JT, Rutledge FN. Immature teratoma of the ovary. Obstet Gynecol 1986; 68: 624-9.

3. Boor PJ, Schoene WC. Fetal cerebellar tissue associated with a primitive neuro-epithelial tumor in an ovarian teratoma. Can J Neurol Sci 1975; 2: 139-41.

4. Kleinman GM, Young RH, Scully RE. Primary neuroectodermal tumors of the ovary: a report of 25 cases. Am J Surg Pathol 1993; 17: 764-78.

5. Talerman A, Vang R. Germ cell tumors of the ovary. In: Kurman RJ, Ellenson LH, Ronnett BM, eds. Blaustein's pathology of female genital tract. 6th ed. New York: Springer, 2011; 847-907.

6. Smith $\mathrm{HO}$, Berwick $\mathrm{M}$, Verschraegen $\mathrm{CF}$, et al. Incidence and survival rates for female malignant germ cell tumors. Obstet Gynecol
2006; 107: 1075-85.

7. Gallion H, van Nagell JR Jr, Donaldson ES, Hanson MB, Powell DF. Immature teratoma of the ovary. Am J Obstet Gynecol 1983; 146: $361-5$

8. Sanes DH, Reh TA, Harris WA. Development of the nervous system. 2nd ed. San Diego: Academic Press, 2006; 57-85.

9. Kierszenbaum AL. Histology and cell biology: an introduction to pathology. St. Louis: Mosby, 2002; 199-225.

10. Ballabh P, Braun A, Nedergaard M. Anatomic analysis of blood vessels in germinal matrix, cerebral cortex, and white matter in developing infants. Pediatr Res 2004; 56: 117-24.

11. Dummula K, Vinukonda G, Xu H, et al. Development of integrins in the vasculature of germinal matrix, cerebral cortex, and white matter of fetuses and premature infants. J Neurosci Res 2010; 88: 1193-204.

12. Sugiyama T, Osumi N, Katsuyama Y. The germinal matrices in the developing dentate gyrus are composed of neuronal progenitors at distinct differentiation stages. Dev Dyn 2013; 242: 1442-53.

13. Baker PM, Rosai J, Young RH. Ovarian teratomas with florid benign vascular proliferation: a distinctive finding associated with the neural component of teratomas that may be confused with a vascular neoplasm. Int J Gynecol Pathol 2002; 21: 16-21.

14. Gaudin PB, Rosai J. Florid vascular proliferation associated with neural and neuroendocrine neoplasms: a diagnostic clue and potential pitfall. Am J Surg Pathol 1995; 19: 642-52. 